\title{
COMMUTATORS IN SEMI-SIMPLE ALGEBRAIC GROUPS
}

\author{
RIMHAK REE
}

Introduction. In [2] S. Pasiencier and H.-C. Wang proved that every element in a complex semi-simple Lie group is a commutator. The purpose of this note is to show that their method can be applied to the case of semi-simple algebraic groups without any restriction on the characteristic of the ground field. We shall prove the following

THEOREM. In a connected semi-simple algebraic group defined over an algebraically closed field, every element is a commutator.

For an additive analogue of the above theorem for semi-simple Lie algebras, see [3], where the algebraic closedness of the ground field is not assumed.

1. Notation and preliminary. We shall use the terminology and results in [1]. Let $G$ be a connected semi-simple algebraic group defined over an algebraically closed field $K, T$ a maximal torus of $G, B$ a Borel subgroup containing $T, B^{u}$ the unipotent part of $B, N$ the normalizer of $T$ in $G, W=N / T$ the Weyl group, and $X=X(T)$ the character group of $T$.

The character group $X$ is a free abelian group of finite rank $n$. The Weyl group $W$ acts on $X$ by

$$
(w \chi)(t)=\chi\left(\omega^{-1} t \omega\right)
$$

where $\omega$ is an element in $N$ representing $w$, and $\chi \in X, t \in T$. Also, $X$ is equipped with a positive definite metric such that $2(\chi, \alpha) /(\alpha, \alpha)$ is an integer for any $\chi \in X$ and any root $\alpha$. We shall normalize the metric such that $(\alpha, \alpha)$ is an even integer for any root $\alpha$, so that $(\chi, \alpha)$ is an integer for any $\chi \in X$ and any root $\alpha$. For any root $\alpha$, the Weyl reflection $w_{\alpha}: X \rightarrow X$ defined by $w_{\alpha}(\chi)=\chi-2(\chi, \alpha)(\alpha, \alpha)^{-1} \alpha$ belongs to $W$, and $W$ is generated by the $w_{\alpha}$.

For each root $\alpha>0$, there is a homomorphism $\tau_{\alpha}: K \rightarrow B^{u}$ of the additive group of $K$ into $B^{u}$. Any element in $B$ can be written uniquely in the form $t \prod_{\alpha} \tau_{\alpha}\left(x_{\alpha}\right)$, where $t \in T, x_{\alpha} \in K$, and where the product runs over all roots $\alpha>0$ in the increasing order. For any $t \in T$ we have $t \tau_{\alpha}(x) t^{-1}=\tau_{\alpha}(\alpha(t) x)$, and the commutator $\left(\tau_{\alpha}(x), \tau_{\beta}(y)\right)$ can be written as a product $\prod \tau_{\gamma}\left(z_{\gamma}\right)$ with $\gamma \geqq \alpha+\beta$. From this we have

(1.2) Let $\alpha_{1}, \alpha_{2}, \cdots, \alpha_{M}$ be all the positive roots in increasing order.

Received by the editors February 12, 1963. 
For $1 \leqq m \leqq M$, denote by $B_{m}^{u}$ the group generated by the $\tau_{\alpha_{i}}(x)$, where $\alpha_{i} \geqq \alpha_{m}$ and $x \in K$. Then $B_{m}^{u}$ is a normal subgroup of $B^{u}$.

2. Main lemmas. An element $t \in T$ is said to be regular if $\alpha(t) \neq 1$ for all roots $\alpha$. As in [2] our theorem follows from the following three lemmas.

(2.1) Every element in $G$ is conjugate to an element in $B$ (cf. [1, Exposé 6, p. 13]).

(2.2) If $t \in T$ is regular, then for any $s \in B^{u}, t$ and $t s$ are conjugate in $B^{u}$.

(2.3) For any $t \in T$ there exists a regular $t_{0} \in T$ and an element $\omega \in N$ such that $\omega^{-1} t_{0} \omega=t_{0} t$.

We shall prove (2.2) as the special case $m=1$ of the following:

$\left(2.2^{\prime}\right)$ Let $\alpha_{m}$ and $B_{m}^{u}$ be as in (1.2). Let $t \in T$ be regular. Then for any given $s \in B_{m}^{u}$ there exists an element $u \in B_{m}^{u}$ such that $u t u^{-1}=t s$.

If $m>M$, then $\left(2.2^{\prime}\right)$ is trivially true. We shall prove $\left(2.2^{\prime}\right)$ by descending induction on $m$. Suppose $m \leqq M$ and that $\left(2.2^{\prime}\right)$ is proved for greater $m$. We can write $s=\tau_{\alpha}(a) s^{\prime}$, where $\alpha=\alpha_{m}$, and $s^{\prime} \in B_{m+1}^{u}$. From the regularity of $t$ we have $\alpha(t) \neq 1$, hence there exists $b \in K$ such that $\alpha(t)^{-1} b=a+b$. By (1.2) the element $s^{\prime \prime}=\tau_{\alpha}(-b) s^{\prime} \tau_{\alpha}(b)$ is clearly in $B_{m+1}^{u}$. Hence by the assumption of induction there exists $u^{\prime} \in B_{m+1}^{u}$ such that $u^{\prime} t u^{\prime-1}=t s^{\prime \prime}$. Now it is easy to verify that $u=\tau_{\alpha}(b) u^{\prime}$ satisfies $u t u^{-1}=t s$. Thus $\left(2.2^{\prime}\right)$ is proved.

3. Proof of (2.3). In order to obtain clarity we shall state a theorem of Kostant used in [2, p. 910].

(3.1) Let $X^{Q}=X \otimes Q$ be the vector space over the field $Q$ of rational numbers derived from $X$ by extending the coefficient domain. For $w \in W$, set $X_{1}^{Q}=\left\{x \in X^{Q} \mid w(x)=x\right\}$ and let $X_{2}^{Q}$ be the orthogonal complement of $X_{1}^{Q}$ in $X^{Q}$. Then $X^{Q}=X_{1}^{Q} \oplus X_{2}^{Q}$, and $X_{2}^{Q}=(w-1) X_{2}^{Q}=\left(w^{-1}-1\right) X_{2}^{Q}$. The subspace $X_{2}^{Q}$ has a basis $\left(\alpha_{1}, \alpha_{2}, \cdots, \alpha_{m}\right)$ consisting of roots such that

$$
w=w_{\alpha_{1}} w_{\alpha_{2}} \cdots w_{\alpha_{m}} .
$$

Conversely, if $w$ is given by (3.1.1) and if $\alpha_{1}, \alpha_{2}, \cdots, \alpha_{m}$ are linearly independent roots, then $\left(\alpha_{1}, \alpha_{2}, \cdots, \alpha_{m}\right)$ is a basis of $X_{2}^{Q}$. If $\alpha$ is a root contained in $X_{2}^{Q}$, then

$$
\operatorname{dim}\left(w_{\alpha} w-1\right) X^{Q}<\operatorname{dim}(w-1) X^{Q} .
$$

Now let $P$ be the set of all $\alpha \in X^{Q}$ such that $(\chi, \alpha)$ is an integer for any $\chi \in X$. Clearly all the roots are in $P$. For any $\alpha \in P$ and $z \in K^{*}$, define $t(\alpha, z) \in T$ by $\chi(t(\alpha, z))=z^{(\chi, \alpha)}$, and denote by $T(\alpha)$ the group of all $t(\alpha, z)$, where $z \in K^{*}$. 
(3.2) If $\alpha \in P$ is a linear combination of $\alpha_{1}, \alpha_{2}, \cdots, \alpha_{m}$ in $P$ with coefficients in 8 , then $T(\alpha) \subseteq T\left(\alpha_{1}\right) T\left(\alpha_{2}\right) \cdots T\left(\alpha_{m}\right)$.

For the proof, let $0 \neq a \in \boldsymbol{Z}$ such that $a \alpha=\sum a_{i} \alpha_{i}$ with $a_{i} \in \boldsymbol{Z}$. For any $z \in K^{*}$, find $x \in K^{*}$ such that $x^{a}=z$. Then

$$
\chi(t(\alpha, z))=z^{(x, \alpha)}=\prod_{i} x^{\left(x, a_{i} \alpha_{i}\right)}=\chi\left(\prod_{i} t\left(\alpha_{i}, x^{a_{i}}\right) .\right.
$$

Hence $t(\alpha, z)=\prod_{i} t\left(\alpha_{i}, x^{a_{i}}\right)$. This proves (3.2).

(3.3) If $\left(\alpha_{1}, \alpha_{2}, \cdots, \alpha_{n}\right)$ is a basis of $X^{Q}$ lying in $P$, then

$$
T=T\left(\alpha_{1}\right) T\left(\alpha_{2}\right) \cdots T\left(\alpha_{n}\right) .
$$

For the proof, let $\left(\chi_{1}, \chi_{2}, \cdots, \chi_{n}\right)$ be a basis of $X$. Then the $n \times n$ matrix $\left(\left(\chi_{i}, \alpha_{j}\right)\right)$ is nonsingular. Since $K$ is algebraically closed, there exist $z_{1}, z_{2}, \cdots, z_{n} \in K^{*}$ such that

$$
\prod_{j} z_{j}\left(x_{i}, \alpha_{j}\right)=\chi_{i}(t) \quad(1 \leqq i \leqq n)
$$

for any given $t \in T$. Then $t=\prod_{j} t\left(\alpha_{j}, z_{j}\right)$. Thus (3.3) is proved.

For any $w \in W$ and $t \in T$ define $t^{w}$ by $t^{w}=\omega^{-1} t \omega$, where $\omega$ is an element in $N$ representing $w$. Set $t^{w-1}=t^{w} t^{-1}$, and denote by $T^{w-1}$ the group of all $t^{w-1}$, where $t \in T$.

(3.4) If $w$ is given by (3.1.1) with linearly independent roots $\alpha_{1}, \alpha_{2}$, $\cdots, \alpha_{m}$, then $T^{w-1}=T\left(\alpha_{1}\right) T\left(\alpha_{2}\right) \cdots T\left(\alpha_{m}\right)$. Any $t \in T^{w-1}$ can be written in the form $t=t^{\prime w-1}$, where $t^{\prime} \in T^{w-1}$.

For any root $\alpha$, we have $\left(w^{-1}-1\right) \alpha=\sum n_{i} \alpha_{i}$ with $n_{i} \in Z$. Hence for any $z \in K^{*}$ and $\chi \in X$, we have

$$
\begin{aligned}
\chi\left(t(\alpha, z)^{w-1}\right) & =z^{(w \chi-\chi, \alpha)}=z^{\left(\chi,\left(w^{-1}-1\right) \alpha\right)} \\
& =\prod_{i} z^{\left(\chi, n_{i} \alpha_{i}\right)}=\chi\left(\prod_{i} t\left(\alpha_{i}, z_{i}\right)\right),
\end{aligned}
$$

where $z_{i}=z^{n_{i}}$. Hence $t(\alpha, z)^{w-1}=\prod_{i} t\left(\alpha_{i}, z_{i}\right)$. Since this is true for all roots $\alpha$ and $z \in K^{*}$, we have, by (3.3), $T^{w-1} \subseteq T\left(\alpha_{1}\right) T\left(\alpha_{2}\right) \cdots T\left(\alpha_{m}\right)$. On the other hand, by (3.1) there exists $\alpha \in P$ such that $\left(w^{-1}-1\right) \alpha$ $=n \alpha_{i}$ with some nonzero integer $n$. Then $t(\alpha, z)^{w-1}=t\left(\alpha_{i}, z^{n}\right)$. Since $z \in K^{*}$ and $i$ are arbitrary, it follows that $T\left(\alpha_{1}\right) T\left(\alpha_{2}\right) \cdots T\left(\alpha_{m}\right)$ $\subseteq T^{w-1}$. Actually, (3.1) implies that for the given $\alpha_{i}$ one can take $\alpha$ in $P \cap X_{2}^{Q}$. Then by (3.2) $t(\alpha, z) \in T\left(\alpha_{1}\right) T\left(\alpha_{2}\right) \cdots T\left(\alpha_{m}\right)=T^{w-1}$. Thus the second part of (3.4) is also proved.

Now we shall prove (2.3). Let $t \in T$ be given. By (3.3) $t \in T\left(\alpha_{1}\right) T\left(\alpha_{2}\right)$ $\cdots T\left(\alpha_{m}\right)$ for some roots $\alpha_{1}, \alpha_{2}, \cdots, \alpha_{m}$, since $X^{Q}$ is spanned by the roots. Let $m$ be the least integer for which this is possible. Then by (3.2) the roots $\alpha_{1}, \alpha_{2}, \cdots, \alpha_{m}$ are linearly independent. Set

$$
w=w_{\alpha_{1}} w_{\alpha_{2}} \cdots w_{\alpha_{m}} \text {. }
$$


Then by (3.4),

$$
t=t^{\prime w-1} \quad \text { with } \quad t^{\prime} \in T\left(\alpha_{1}\right) T\left(\alpha_{2}\right) \cdots T\left(\alpha_{m}\right) .
$$

We shall show that

$$
\alpha\left(t^{\prime}\right) \neq 1
$$

for any root $\alpha$ contained in $(w-1) X^{Q}$. Suppose the contrary. Then by (1.1)

$$
\begin{aligned}
\chi\left(t^{\prime w_{\alpha} w-1}\right) & =\left(w_{\alpha}(w \chi)-\chi\right) t^{\prime} \\
& =\left(w \chi-\chi-2(\chi, \alpha)(\alpha, \alpha)^{-1} \alpha\right) t^{\prime} \\
& =(w \chi-\chi) t^{\prime}=\chi\left(t^{\prime w-1}\right)=\chi(t)
\end{aligned}
$$

for all $\chi \in X$. Hence $t=t^{\prime w^{\prime}-1}$, where we put $w^{\prime}=w_{\alpha} w$. By (3.1), $m^{\prime}=\operatorname{dim}\left(w^{\prime}-1\right) X^{Q}<\operatorname{dim}(w-1) X^{Q}=m$. Hence by (3.1) and (3.4), $T^{w^{\prime}-1}=T\left(\beta_{1}\right) T\left(\beta_{2}\right) \cdots T\left(\beta_{m^{\prime}}\right)$ for some roots $\beta_{1}, \beta_{2}, \cdots, \beta_{m^{\prime}}$. But $t=t^{\prime w^{\prime}-1} \in T^{w^{\prime}-1}$. This contradicts the minimality of $m$. Thus (3.6) is proved.

For the element $w$, let $X^{Q}=X_{1}^{Q} \oplus X_{2}^{Q}$ be the decomposition given in (3.1). We have $(w-1) X^{Q}=X_{2}^{Q}$. Let $\gamma_{1}, \gamma_{2}, \cdots, \gamma_{k}$ be all the roots which are not in $X_{2}^{Q}$, and let $\gamma_{i}=\gamma_{i}^{\prime}+\gamma_{i}^{\prime \prime}$, where $\gamma_{i}^{\prime} \in X_{1}^{Q}, \gamma_{i}^{\prime \prime} \in X_{2}^{Q}$. We shall show that if $z_{1}, z_{2}, \cdots, z_{k} \in K^{*}$ are suitably chosen and if we set $t^{\prime \prime}=\prod t\left(c \gamma_{i}^{\prime}, z_{i}\right)$ with a nonzero integer $c$ such that $c \gamma_{i}^{\prime} \in P$ for $1 \leqq i \leqq k$, then $t_{0}=t^{\prime} t^{\prime \prime}$ is the desired element. It is clear that $t^{\prime \prime w-1}=1$. Hence by (3.5), $t_{0}^{20-1}=t$. Also for any $\operatorname{root} \alpha$ in $X_{2}^{Q}, \alpha\left(t^{\prime \prime}\right)=1$, hence by (3.6) $\alpha\left(t_{0}\right) \neq 1$. Now for the roots $\gamma_{i}, 1 \leqq i \leqq k$, we have

$$
\gamma_{i}\left(t_{0}\right)=\gamma_{i}\left(t^{\prime}\right) \prod_{j} z_{j}^{\left(\gamma_{i}, c \gamma_{j^{\prime}}\right)}
$$

Since $\gamma_{i}$ is not in $X_{2}^{Q}$, for each $\gamma_{i}$ there exists an index $j$ such that $\left(\gamma_{i}, c \gamma_{j}\right) \neq 0$. Since $K$ has infinitely many elements, one can take $z_{1}, z_{2}, \cdots, z_{k} \in K^{*}$ such that $\gamma_{i}\left(t_{0}\right) \neq 1$ for $1 \leqq i \leqq k$. We have $t=t_{0}^{t o-1}$, or $\omega^{-1} t_{0} \omega=t t_{0}$. Thus (2.3) is proved.

\section{REFERENCES}

1. C. Chevalley, Classification des groupes de Lie algébriques, Séminaire, École Normale Supérieure, Paris, 1956-1958.

2. S. Pasiencier and H.-C. Wang, Commutators in a complex semi-simple Lie group, Proc. Amer. Math. Soc. 13 (1962), 907-913.

3. Gordon Brown, On commutators in a simple Lie algebra, Proc. Amer. Math. Soc. 14 (1963), 763-767.

University of British Columbia, Vancouver, B. C., Canada 